Manuscript for Regular paper: JELECHEM-D-07-00602 1st revision

J. Electroanal. Chem.

\title{
Formation of viologen radical cation condensed phase through two-dimensional molecular organization process on an HOPG electrode surface in binary viologen solutions
}

\author{
Yasuhiko Tanaka $^{\mathrm{a}}$ and Takamasa Sagara ${ }^{\mathrm{b}, *}$ \\ ${ }^{a}$ Department of Materials Science, Graduate School of Science and Technology, \\ Nagasaki University, Bunkyo 1-14, Nagasaki 852-8521, Japan \\ ${ }^{b}$ Department of Applied Chemistry, Faculty of Engineering, Nagasaki University, \\ Bunkyo 1-14, Nagasaki 852-8521, Japan
}

Received 17 October 2007; received in revised form

Footnote

*Corresponding author. Tel. +81-95-819-2676; fax: +81-95-819-2675

E-mail address: sagara@nagasaki-u.ac.jp (T. Sagara)

${ }^{a}$ Present affiliation: Department of Applied Chemistry, Graduate School of Engineering, Kyushu University, Motooka 744, Nishi-ku, Fukuoka, 819-0395, Japan 


\begin{abstract}
Viologens possessing long alkyl chains are condensed to monolayers of their radical cations upon one-electron reduction on a basal plane of a highly-oriented pyrolytic graphite (HOPG) electrode in contact with not only one-component viologen aqueous solutions but also binary mixtures. For three types of binary mixtures of viologen including ten different combinations, the condensed phase formation processes were described using the results of voltammetric measurements. First, in the case of the binary mixture of symmetric di-alkyl viologens $(d A)$ with different chain lengths, two-dimensional (2D) phase separation into two domains took place in the course of cathodic potential scan in a certain range of the solution molar fraction when the chain length difference is two methylene units or more. Second, in the case of the mixture of a $d \mathrm{~A}$ and its both-end carboxylated derivative (bis-carboxylated viologen: $b \mathrm{C}$ ), either pure $b \mathrm{C}$ condensed phase or $d \mathrm{~A}+b \mathrm{C}$ well-mixed phase covered the entire surface area. Third, in the case of the mixture of $d \mathrm{~A}$ and $b \mathrm{C}$ with far different chain lengths, the molar fraction dependent condensation processes took place while $b \mathrm{C}$ always dominates the phase formation behavior. The implications of these results were discussed as typical 2D molecular nano-organization processes in the terms of homo- and hetero-intermolecular interactions on the HOPG surface.
\end{abstract}

Keywords: Phase transition; HOPG electrode; Di-alkyl viologen; Bis-carboxylated viologen; Binary mixture 


\section{Introduction}

It is presently one of the central issues of chemistry to provide the basis of accurate means to control various molecular organization structures. In molecular organization chemistry, design and quantitative evaluation of homo- and hetero-intermolecular interactions are of importance. When the molecules organize into crystals, liquid-crystals, self-assembled structures, and two-dimensionally aligned structures, interaction with neighboring molecules is usually the dominant factor over long range interactions. As well as the clarification of the relationship between the structures of individual molecules and organized assemblies, understanding of dynamics of both formation and dissolution processes is indispensable to shed light in depth on the molecular organization.

The use of a molecular organization process to form a monolayer driven by potential control at an electrified interface is well-suited to describe the factors governing the organization process [1-3]. Among others, the merit of the observation of such processes on an electrode surface includes: (1) macroscopic data such as voltammograms sensitively reflect the monolayer structures and formation kinetics, because voltammetric current directly represents the amount of the molecules in the monolayer and potential dependent dynamics, (2) the fine control of the potential can precisely switch the states of the molecule as well as the micro-environment of the interface, and (3) reduction of the space dimension from three-dimension (3D) in a homogeneous medium to $2 \mathrm{D}$ at a heterogeneous interface enables us to focus our attention on the lateral intermolecular interaction as a determinant factor, though interaction with an electrode surface and solvation/desolvation effect should be additionally taken into account.

When a monomolecular film on an electrode surface exhibits the first-order phase transition between condensed and diffuse phases in response to the potential change, the transition process appears as sharp voltammetric peaks [3]. Examples described in recent reports include both nonfaradaic transitions [4-7] and faradaic ones [8-12]. The 
voltammetric peak position, wave-shape, and dependence on the time-scale of the experimental parameters, such as a potential scan rate, tell us a plenty of information of thermodynamics and kinetics.

Viologen molecules with long alkyl chains exhibit the first-order 2D phase transition upon their one-electron redox reactions between viologen dication $\left(\mathrm{V}^{2+}\right)$ and viologen radical cation $\left(\mathrm{V}^{\bullet+}\right)$ on a basal plane of a highly-oriented pyrolytic graphite (HOPG) electrode $[10,11,13-16]$. The transition takes place between a gas-like adsorption state of $\mathrm{V}^{2+}$ and a $2 \mathrm{D}$ condensed monolayer of $\mathrm{V}^{\bullet+}$. The cathodic condensation process is a typical 2D molecular organization. In our previous studies, the potential separation between cathodic and anodic spike-like voltammetric peaks, corresponding respectively to condensation and dissolution processes, was found to reflect the strength of the lateral intermolecular interaction. The interaction in the condensed monolayer is of multiple modes. It includes $\pi-\pi$ stacking of viologen cation radical rings, which are oriented in side-on configuration on the HOPG electrode surface, and chain-chain interaction between alkyl chains, which are lying flat. When the alkyl chain end is carboxylated, intermolecular hydrogen bonding interaction contributes largely as a lateral interaction in an acidic medium $[15,17]$.

The occurrence of the phase transition is not restricted to a single component viologen system. A binary mixture of viologens also exhibits phase transition on an HOPG electrode surface as demonstrated in our previous paper [18]. In general, in the single component systems, intermolecular homo-interaction is only the factor to be considered in addition to the interaction with the electrode surface and the interplay of solvent. In the binary systems, intermolecular hetero-interaction joins the important factors. Elucidation of interplay of these interaction modes, together with the molecule-surface interactions, is a fundamental issue to understand the $2 \mathrm{D}$ molecular organization processes at a molecular level [19-27]. The 2D molecular organization processes of viologen binary systems may provide us with the opportunity to shed light in depth on the interplay of interactions.

The advantageous features of the use of an HOPG electrode|viologen solution 
interface for the study of phase transition processes of binary systems accompanying 2D molecular organization include: (1) not only the dissolution process of the condensed phase but also the formation process can be directly monitored by electrochemical methods, (2) because the double-layer charging is predominantly due to the space-charge layer charging of the HOPG surface [28,29], non-faradaic current is small and insensitive to the potential as well as to the presence of an organic thin film. In contrast, in the case of reductive desorption of a self-assembled monolayer (SAM) of alkanethiol or its derivative at a Au electrode (this process can be also regarded as a typical 2D phase transition process), only the dissolution process gives rise to the response of significance $[19,21,30]$. In addition, double-layer charging of solution side, which is largely dependent on potential and the presence of superficial films, should always be properly taken into consideration on a metal electrode [31].

The aim of this work is at the elucidation of the conjugation of homo- and hetero-intermolecular interactions in the electrochemically triggered 2D molecular organization processes to condense to a monolayer. We describe the condensation process in the $2 \mathrm{D}$ phase transition of binary viologens on an HOPG electrode surface using the results of the measurements of cyclic voltammograms (CVs).

\section{Scheme 1}

We use six different viologen molecules including four symmetric di-alkyl viologens $(d \mathrm{~A}-m)$ and two bis-carboxylated viologens $(b \mathrm{C}-n)$, and ten binary combinations (Scheme 1). Among the combinations examined, description for $b \mathrm{C}-4+$ bC-7 mixture should be omitted, because the overlap of bulk reduction of $b \mathrm{C}-7$ at $-0.56 \mathrm{~V}$ (vs. $E_{\mathrm{Ag}|\mathrm{AgCl}| \mathrm{sat} \text { 'd KCl})}$ with cathodic phase transition of $b \mathrm{C}-4$ at $-0.57 \mathrm{~V}$ gave rise to complication, avoiding our straightforward analysis.

The mixing combinations of two different viologens can be classified into three types.

Type 1 (solid line in Scheme 1) is the binary mixture of $d$ As. The alkyl chain 
length and its difference may determine chain-chain van der Waals interaction, solubility, sequence of condensation, and steric mismatch. The effect of these factors on the behavior of binary systems should be elucidated. In this type, the behavior for the combination of $d \mathrm{~A}-4+d \mathrm{~A}-7$ has already been described in detail in our previous communication [18]: the formation of two separated domains of condensed phases takes place in a certain range of mixing ratio in the course of cathodic potential scan. It was surprising that $d \mathrm{~A}-7$, which has less negative cathodic transition potential and thus is given the preceding opportunity to condense in the course of the cathodic potential scan, does not cover the entire surface with its condensed phase but leaves a free HOPG surface area behind, allowing subsequent condensation of $d \mathrm{~A}-4$ at more negative potentials. The phase separated state, however, was found to be a transitory one so that the finally reached state at very negative potentials is the thermodynamically most stable single component phase of $d \mathrm{~A}-7$.

Type 2 (double solid lines in scheme 1) is the binary mixture of $d \mathrm{~A}$ and $b \mathrm{C}$, where the number of methylene units of the latter is the same as the former if including the end methyl group. The question to be answered is how the two distinct terminal groups affect the condensation. The terminal carboxylic acid group has an ability to form hydrogen bonding network of $b \mathrm{C}$ molecules on a hydrophobic HOPG surface in acidic aqueous medium $[15,17]$. The behavior of the combination of $d \mathrm{~A}-7+b \mathrm{C}-7$ has been described in detail in our previous communication [18]: two molecules are well mixed in a condensed phase.

Type 3 (broken lines in scheme 1 ) is the binary mixture of $d \mathrm{~A}$ and $b \mathrm{C}$ with different numbers of methylene units.

It is out of the scope of this paper to elucidate the detailed molecular alignment structure and phase formation kinetics for each of specific mixing combination of two viologens. With an aim at overviewing the perspective of the phase transition processes, we use herein a common measurement condition in the voltammetric studies. Constant start potential and scan rate of the potential scan in CV measurements and total concentration of the two coexisting components were employed unless otherwise stated. 
Overall, 2D compatibility of the two components and the effect of the presence of the terminal interactive group are highlighted.

\section{Experimental Section}

Except for heptylviologen (dA-7), which was recrystallized from commercial reagent $(\mathrm{TCI}), d \mathrm{~A}$ molecules were prepared by a Menshutkin reaction of sublimated 4,4'-bipyridine with corresponding alkylbromide in DMF. 1,1'-bis(7-carboxyheptyl)-4,4'-bipyridinium dibromide (bC-7) was prepared in our previous study [15]. 1,1'-bis(4-carboxybutyl)-4,4'-bipyridinium dibromide (bC-4) was synthesized as the similar manner as $b C-7$. Water was purified through a Milli-Q Plus Ultrapure water system coupled with an Elix-5 kit (Millipore Co.). Its resistivity was over $18 \mathrm{M} \Omega \mathrm{cm}$. All other chemicals were of reagent grade and used as received. An HOPG plate was the product of Matsushita Electric Co. (Panasonic graphite, PGX04 or PGX05: size $12 \times 12 \times 3 \mathrm{~mm}$-thickness). The plate was connected perpendicularly to a copper pipe. To expose a fresh basal plane, the surface of the HOPG was peeled off using a Scotch adhesive tape immediately before use. The HOPG electrode (electrode area: $A=1.44 \mathrm{~cm}^{2}$ ) was horizontally touched to the Ar gas/viologen solution interface and set in a hanging meniscus $(\mathrm{H}-\mathrm{M})$ configuration in an electrochemical cell. Note that the first touch of the HOPG electrode to the gas/solution interface followed by voltammetric measurements is the prerequisite to realize sharp transition responses [11]. Therefore, whenever changing the solution phase composition, the peeling-off and H-M touching procedures were conducted. All the electrochemical measurements were made using a $\mathrm{Ag}|\mathrm{AgCl}|$ saturated $\mathrm{KCl}$ reference electrode and a coiled $\mathrm{Au}$ wire counter electrode under an Ar gas (>99.998\%) atmosphere. For the voltammetric measurements, a potentiostat (HUSOU, HECS-9094) coupled with a function generator (HUSOU, HECS-321B) was employed. All the measurements were carried out at $23 \pm$ $1^{\circ} \mathrm{C}$. Unless otherwise stated, base electrolyte solution was $0.3 \mathrm{M} \mathrm{KBr}$ acidified to $\mathrm{pH}$ 
2.3 by addition of $\mathrm{HBr}$ in order to keep complete protonation to carboxylate groups of $b C s$. The total concentration of viologen was kept $0.1 \mathrm{mM}$ in all the measurements. When alkaline solution was necessary, $\mathrm{KOH}$ was used to adjust $\mathrm{pH}$. The start potential of the potential scan in $\mathrm{CV}$ measurements was fixed at $0.0 \mathrm{~V}$, where a pre-equilibration time of 5 min was given, unless otherwise stated.

\section{Results}

Table 1

In order to be consistent with our previous work [18] in regards to experimental conditions, we used commonly $80 \mathrm{mV} \mathrm{s}^{-1}$ as a standard potential scan rate for $\mathrm{CV}$ measurements. For the sake of the analysis of CVs, we first list in Table 1 the anodic and cathodic transition peak potentials ( $E_{\mathrm{pa}}$ and $E_{\mathrm{pc}}$, respectively) of CVs at $80 \mathrm{mV} \mathrm{s}^{-1}$, together with the midpoint potential, $E_{1 / 2, \mathrm{a}}=\left(E_{\mathrm{pa}}+E_{\mathrm{pc}}\right) / 2$, and the peak separation, $\Delta E_{\mathrm{p}}$ $=E_{\mathrm{pa}}-E_{\mathrm{pc}}$, for all six viologens used. Those values will be referred to when necessary.

Note that CVs were frequently dependent of both the start potential and pre-equilibration time. For example, the cathodic peak of bC-4 (see 3.2) exhibited slightly different wave shape when the start potential was set far more positive than $0.0 \mathrm{~V}$. To avoid complexity, for example due to partial surface oxidation of HOPG, we fixed the start potential at $0.0 \mathrm{~V}$ in the present work. In the binary mixture of $d \mathrm{~A}-7$ and dA-4 (see 3.1.3), when the pre-equilibration time at $0.0 \mathrm{~V}$ was short, the more positive redox couple appeared smaller. Most likely, it takes time to establish equilibrium state of gas-like adsorption layer of oxidized forms at $0.0 \mathrm{~V}$. For all the systems, however, whenever we used a pre-equilibration time longer than $5 \mathrm{~min}$, we obtained constant CVs. Therefore, for all the CVs discussed herein, a constant start potential at $0.0 \mathrm{~V}$ and pre-equilibration time of $5 \mathrm{~min}$ were used. We are underway of detailed study on what 
is occurring during the pre-equilibration time, and the results will be reported elsewhere.

\subsection{Mixture of two symmetrical di-alkyl viologens (dAs) with different chain lengths}

The CVs of the series of $d$ As for their single component cases at an HOPG electrode were previously reported by Arihara and coworkers [10]. They reported that, with increasing the alkyl chain length from $d \mathrm{~A}-2$ to $d \mathrm{~A}-8, E_{1 / 2, \mathrm{a}}$ shifts to less negative and $\Delta E_{\mathrm{p}}$ becomes greater. Our results in the present work (Table 1) agreed well with theirs. The increase in adsorptivity due to stronger attractive interaction of longer alkyl chain to the hydrophobic HOPG surface shifts $E_{1 / 2, \mathrm{a}}$ to less negative. The decrease in solubility of reduced form with the increase of the chain length also drives the shift of $E_{1 / 2, \mathrm{a}}$ to less negative. The increase of intermolecular chain-chain interaction in the condensed phase of radical cations with longer alkyl chains leads to the increase in $\Delta E_{\mathrm{p}}$.

Fig. 1

\subsubsection{Mixture of $d A-m+d A-(m-1)$ for $m=5,6$, and 7}

Typical results of CV measurements for the title case, where the difference of the alkyl chain length is one methylene $\left(-\mathrm{CH}_{2}\right)$ unit, are shown in Fig. 1. The dependence of voltammetric wave on the molar fraction $\left(x_{\mathrm{m}}\right.$ : where subscript $m$ stands for the alkyl chain length of the longer viologen) was principally common for $m=5,6$ and 7 . One couple of anodic and cathodic transition peaks was always observed regardless of $x_{\mathrm{m}}$, indicating well-mixing of two viologen molecules in the condensed phase. The difference of one methylene unit length did not induce molecular alignment disorder even for the shortest chain combination of $d \mathrm{~A}-4$ and 5 in the present case, showing sharp transition peaks in CVs. The value of $E_{1 / 2, a}$ changed monotonically with the change of $x_{\mathrm{m}}$.

Fig. 2 
Fig. 2 represents $x_{6}$ dependence of $E_{\mathrm{pa}}$ and $E_{\mathrm{pc}}$ for a mixture of $d \mathrm{~A}-6+d \mathrm{~A}-5$. The non-linear dependence of $E_{1 / 2, \mathrm{a}}$ on $x_{6}$, in particular the tendency of the convexity of the curve toward the $E_{1 / 2, \mathrm{a}}$ value of the longer chain viologen ( $\left.d \mathrm{~A}-6\right)$, reveals that longer one is dominantly involved in the condensed phase compared to the shorter counterpart. This indicates that the molar fraction of the longer chain viologen in the mixed condensed phase is greater than the molar fraction in solution phase. This curvature was also observed for the mixtures of $d \mathrm{~A}-5+d \mathrm{~A}-4$ and $d \mathrm{~A}-7+d \mathrm{~A}-6 . \quad$ In the case of an ideal or regular mixture of two different redox species confined on an electrode surface with the identical molar fractions to the solution composition, the half-wave potential is symmetrically shifted from the simple linear weighted mean with respect to the solution composition due to the entropy of mixing, and the maximum deviation is $c a .18 \mathrm{mV}$ at 1:1 solution composition for the ideal mixture on the electrode surface $[32,33]$. The observed curvature reflecting an asymmetric shift (Fig. 2) rationalizes above interpretation.

The well-mixing into a unique binary phase has frequently been observed, for example, for the SAMs of two alkanethiol molecules with a minor difference of alkyl chain length on a Au electrode [21].

Fig. 3

\subsubsection{Mixture of $d A-m+d A-(m-2)$ for $m=6$ and 7}

Two combinations with the difference of the alkyl chain length of two methylene units, $d \mathrm{~A}-6+d \mathrm{~A}-4$ and $d \mathrm{~A}-7+d \mathrm{~A}-5$, are concerned (Fig. 3). For $d \mathrm{~A}-6+d \mathrm{~A}-4$, formation of nearly pure phase of $d \mathrm{~A}-4$ occurred while $x_{6}$ is smaller than 0.01 . However, when $x_{6}$ exceeded $c a$. 0.015 , new domain appeared whose $E_{1 / 2, \mathrm{a}}$ was in between the $E_{1 / 2, a}$ values of individual components. This new phase separated domain consisted of presumably the mixed phase of $d \mathrm{~A}-6$ and $d \mathrm{~A}-4$, in which the content of 
$d$ A-4 as the minor component was decreased with an increase of $x_{6}$. In the range of $x_{6}$ from 0.059 to 0.50 , only one couple of peaks was observed, whereas the peak shape was largely distorted. At higher $x_{6}, \mathrm{CV}$ gradually converged to that of $d \mathrm{~A}-6$ singular system. The tendency of $x_{\mathrm{m}}$ dependent change of CV was nearly the same for $d \mathrm{~A}-7+$ dA-5 mixture. The apparent ranges of $x_{\mathrm{m}}$ exhibiting the coexistences of two phase separated domains were $x_{6}=0.016-0.038$ for $d \mathrm{~A}-6+d \mathrm{~A}-4$ mixture and $x_{7}=$ 0.0099-0.091 for $d A-7+d A-5$. Similar phase separation due to the alkyl chain length difference has been demonstrated by a Monte-Carlo calculation by Shevade and coworkers [34]. Importantly, it was found that the phase separated state observed in $\mathrm{CVs}$ for these two combinations is of transient one as in the case of the $d \mathrm{~A}-7+d \mathrm{~A}-4$ mixture as we reported previously [18]. Potential holding at more negative potential than the potential of more negative couple in the course of the potential scan resulted in the annihilation of the more negative anodic peak and the growth and positive shift of the less-negative anodic peak. Therefore, the phase separated state is transitory and the finally reached state is the one component phase of the viologen molecule with longer chain. The longer alkyl chain viologen has stronger affinity to the HOPG surface and also exhibits stronger homo-intermolecular interaction than shorter one.

\subsubsection{Mixture of $d A-m+d A-(m-3)$ for $m=7$}

The behavior of this mixture was previously reported by us (Fig. 3 in ref. [18]). In short, $x_{7}$ dependency is similar to the cases of $\Delta m=2$. The two phase coexsistence range of $x_{7}$ was from 0.025 to 0.062 , being narrower than that for $d \mathrm{~A}-5+d \mathrm{~A}-7$ mixture but wider than that for $d \mathrm{~A}-4+d \mathrm{~A}-6$ mixture.

\subsection{Behavior of $b C-4$}

It is worthwhile to describe the one-component system of $b C-4$, in comparison with $d \mathrm{~A}-4$, before mixing it with others.

Fig. 4 
In Fig. 4, CVs for singular systems of $b \mathrm{C}-4$ (line A-a) and $d \mathrm{~A}-4$ (line A-1) are found. The value of $\Delta E_{\mathrm{p}}$ for $b \mathrm{C}-4$ obtained from $\mathrm{CV}$ at $0.4 \mathrm{mV} \mathrm{s}^{-1}$ was $46 \mathrm{mV}$. This value was $42 \mathrm{mV}$ greater than that for $d \mathrm{~A}-4\left(\Delta E_{\mathrm{p}}=4 \mathrm{mV}\right.$ with $E_{\mathrm{a}, 1 / 2}=-431 \mathrm{mV}$ at $\left.0.4 \mathrm{mV} \mathrm{s}^{-1}\right)$, indicating significant contribution of the hydrogen bonding interaction between the molecules. In contrast, $\Delta E_{\mathrm{p}}$ at $0.4 \mathrm{mV} \mathrm{s}^{-1}$ for $b \mathrm{C}-7$ was $109 \mathrm{mV}$ greater than that for $d \mathrm{~A}-7$ as reported previously [17]. The increase of $\Delta E_{\mathrm{p}}$ by introducing carboxylic acid groups at both ends corresponds to the widening of the bistable potential region due to the formation of hydrogen bonding with two neighboring molecules on an HOPG surface. Therefore, these results point to the difference of hydrogen bonding interaction energy between 4-carboxybutyl and 7-carboxyhexyl side chains. Whether this difference reflects the alkyl chain length or even-odd number of methylenes remains unsolved.

The values of $E_{\mathrm{a}, 1 / 2}$ were -525 and $-463 \mathrm{mV}$ at $0.4 \mathrm{mV} \mathrm{s}^{-1}$, respectively, for $b C-4$ and $b C-7$. This difference is most likely due to much higher solubility and lower attraction with the HOPG surface for $b \mathrm{C}-4$ than $b \mathrm{C}-7$.

\subsection{Mixture of $d A-m$ and $b C-n$ for $m=n=4$ and $m=n=7$}

The behavior of the mixture of $d \mathrm{~A}-4+b \mathrm{C}-4$ is first concerned, and then it is compared with the behavior of the mixture of $d \mathrm{~A}-7+b \mathrm{C}-7$, which has already been discussed in our previous paper [18].

Fig. 4-A shows a series of CVs for the mixture of $d \mathrm{~A}-4+b \mathrm{C}-4$ with various molar fractions of $d \mathrm{~A}-4\left(x_{\mathrm{A} 4}\right)$. The plots of the peak potentials against $x_{\mathrm{A} 4}$ were shown in Fig. 4-B. In the range of $x_{\mathrm{A} 4} \leq 0.50$, only the response of $b C-4$ was observed, indicative of preferential formation of the singular condensed phase of $b C-4$. When $x_{\mathrm{A} 4}=0.61$ was reached, another pair of peaks appeared overlapped at slightly less negative to the peaks of $b C-4$ singular phase. In the range of $x_{\mathrm{A} 4}=0.80-0.909$, although a small cathodic peak corresponding to $b \mathrm{C}-4$ singular phase formation remained, its anodic counterpart peak was shifted to less negative with broadening. At $x_{\mathrm{A} 4}=0.9975$, no trace of $b C-4$ 
singular phase formation was observed. However, even at this $x_{\mathrm{A} 4}$ value, what was formed was not the pure phase of $d \mathrm{~A}-4$, as being indicated by slightly more negative values of $E_{\mathrm{pc}}$ and $E_{\mathrm{pa}}$ than those of the pure phase. Bis-carboxylated viologen cannot allow contamination with di-alkyl counterpart in its condensed phase, exhibiting no change of peak positions (Fig. 4-B, $x_{\mathrm{A} 4} \geq 0.6$ ). In contrast, a di-alkyl viologen enriched phase can allow contamination with bis-carboxylated one.

The $x_{\mathrm{A} 4}$ dependent change of CVs described above is similar as a whole to $x_{\mathrm{A} 7}$ dependent change of CVs for the mixture of $d \mathrm{~A}-7+b \mathrm{C}-7$ (Fig. 4-C) [18].

\subsection{Cross Mixture of $d A+b C$}

Fig. 5

\subsubsection{Mixture of $d A-7+b C-4$}

A series of typical CVs for the mixture of $d \mathrm{~A}-7+b \mathrm{C}-4$ are shown in Fig. 5-A, together with the relationship between peak potentials and $\log x_{\mathrm{A} 7}$ (Fig. 5-B). In the range of $x_{\mathrm{A} 7} \leq 0.0025$, the condensed phase of $b C-4$ was observed without contamination with $d \mathrm{~A}-7$ in it. In the range of $0.004 \leq x_{\mathrm{A} 7} \leq 0.10$, coexistence of a $b \mathrm{C}-4$ phase and a $d \mathrm{~A}-7$ enriched phase occurred. Around $x_{\mathrm{A} 7}=0.024$, the voltammetric charges corresponding to these two phases were almost the same. When $x_{\mathrm{A} 7} \geq 0.14$, the $d \mathrm{~A}-7$ enriched phase predominantly covered the electrode surface, while it did not appear as a pure $d \mathrm{~A}-7$ phase even at $x_{\mathrm{A} 7}=0.50$ as seen by more negative peak potentials compared to the singular phase of $d \mathrm{~A}-7$. The total peak charge obtained by integrating a plot of the voltammetric current as a function of time after non-faradaic background subtraction was $15 \pm 1 \mu \mathrm{C} \mathrm{cm}^{-2}$, regardless of $x_{\mathrm{A} 7}$, for both anodic and cathodic sides. This fact may reflect the almost the same area occupied by one molecule based on the CPK model (ca. $0.96 \mathrm{~nm}^{2} /$ molecule) for $b \mathrm{C}-4$ and $d \mathrm{~A}-7$. This in turn reveals that, regardless of solution molar fraction, the electrode surface is fully covered by either or both $b \mathrm{C}-4$ and $d \mathrm{~A}-7$ when the potential was scanned to $-0.60 \mathrm{~V}$. 
This fact is in sharp contrast to the case of two phase coexistence in two different $d \mathrm{~A}$ molecule mixture. In the range of $0.0063 \leq x_{\mathrm{A} 7} \leq 0.091$ in Fig. 5, $d \mathrm{~A}-7$ molecules, which were given first coming chance to condense on the electrode surface at less negative potential prior to $b \mathrm{C}-4$, did not cover the entire HOPG electrode surface. And then, $b \mathrm{C}-4$ covers the remaining area on the electrode as its condensed phase.

Fig. 6

\subsubsection{Mixture of $d A-4+b C-7$}

Fig. 6 shows a series of CVs (A) and $x_{\mathrm{A} 4}$ dependent peak potentials (B) for the mixtures of $d \mathrm{~A}-4+b \mathrm{C}-7$. In this combination, both $E_{\mathrm{pa}}$ and $E_{\mathrm{pc}}$ for $d \mathrm{~A}-4$ were in between $E_{\mathrm{pa}}$ and $E_{\mathrm{pc}}$ for $b \mathrm{C}-7$ (see also Table 1), in contrast to $d \mathrm{~A}-7+b \mathrm{C}-4$ combination. When $x_{\mathrm{A} 4}<0.91$, only the phase transition response of $b \mathrm{C}-7$ was observed, whereas peak broadening and appearance of double peak feature of cathodic peak were apparent as $x_{\mathrm{A} 4}$ approached to 0.91. In the range of $0.941 \leq x_{\mathrm{A} 4} \leq 0.976$ in Fig. 6-A, an anodic peak around $-0.44 \mathrm{~V}$ was observed. Its peak potential was near to $E_{\mathrm{pa}}$ of $d \mathrm{~A}-4$ singular system $(-0.43 \mathrm{~V})$. However, corresponding cathodic counterpart peak was not found in the CV. It is likely that, in this range of $x_{\mathrm{A} 4}, d \mathrm{~A}-4$ molecules could not start growth of nuclei by their own. The start of the condensation of $b \mathrm{C}-7$ at $-0.545 \mathrm{~V}$ at the cathodic peak triggered the partial formation of $d \mathrm{~A}-4$ enriched phase, which was reoxidized and dissolved at the new anodic peak in the course of anodic potential scan. This cathodic peak potential $(-0.545 \mathrm{~V})$ was approximately $20 \mathrm{mV}$ more negative than the singular system of $b \mathrm{C}-7$, presumably due to the need to simultaneously initiate the condensation to form two phases. With the increase of $x_{\mathrm{A} 4}$ from 0.962 to 0.9901 , the less negative anodic peak rapidly shifted to more negative and merged into a single peak. At $x_{\mathrm{A} 4}=0.996$, because the charge of the anodic peak was equivalent to the sum of the charges of two cathodic peaks, both $d \mathrm{~A}-4$ and $b \mathrm{C}-7$ condensed at the two cathodic peaks were reoxidized and dissolved simultaneously at one anodic peak. It is likely that sequencially formed two condensed phases merged into a homogenous phase during the 
cathodic scan, which was finally dissolved at the anodic peak. In sharp contrast to the $d \mathrm{~A}-7+b \mathrm{C}-4$ case, compatibility of reduced forms of $d \mathrm{~A}-4+b \mathrm{C}-7$ on the HOPG surface may be high (this interpretation will be discussed in section 4). At $x_{\mathrm{A} 4} \geq 0.9901, d \mathrm{~A}-4$ became able to condense by its own as indicated by the appearance of the cathodic peak at $-0.45 \mathrm{~V}$.

\subsection{In alkaline solution}

It is useful to examine the behavior of the mixtures containing $b \mathrm{C}$ in alkaline solution in which carboxylic acid groups are deprotonated to be carboxylates. It is important to see how the loss of hydrogen bonding formation ability affects the phase transition.

For 1:1 mixture of $d \mathrm{~A}-4+b \mathrm{C}-4\left(x_{\mathrm{A} 4}=0.5\right)$, the $\mathrm{CV}$ curve at $\mathrm{pH} 12.3$ was the same as the $\mathrm{CV}$ of $d \mathrm{~A}-4$ singular phase. When being deprotonated, $b \mathrm{C}-4$ had no chance even to influence with the $d \mathrm{~A}-4$ phase transition.

For $1: 1$ mixture of $d \mathrm{~A}-4+b \mathrm{C}-7\left(x_{\mathrm{A} 4}=0.5\right)$, the $\mathrm{CV}$ curve at $\mathrm{pH} 12.3$ showed only one couple of anodic and cathodic peaks. The peak potentials were near to those of $d \mathrm{~A}-4$ rather than those of $b \mathrm{C}-7$, whereas the difference from those of pure $d \mathrm{~A}-4$ phase was apparent. Most likely, the deprotonated $b \mathrm{C}-7$ molecules were still present in the condensed film enriched with $d \mathrm{~A}-4$. Although the deprotonation resulted in not only the loss of hydrogen bonding ability but also increments of solubility in water and electrostatic repulsion, its longer alkyl chain allowed for participation of $b C-7$ into condensation process.

\section{Discussion}

It is worthwhile first to summarize the main results for three types of binary mixtures. 
Fig. 7

In type 1 (mixture of two different $d A s$ ), appearance of the molar fraction region, in which phase separation into two domains occurs, requires a chain length difference of two or more methylene units (Fig. 3). Otherwise, two dAs form one well-mixed condensed phase at any molar ratio (Fig. 1 and Fig. 7-A). In the condensed phase, the content ratio of the longer alkyl chain $d \mathrm{~A}$ is greater than that in the solution phase. The phase separation, however, is always of a transitory state as long as examined combinations are concerned. When phase separation occurs, potential holding at sufficiently negative potential for a sufficient time period always leads to a full surface coverage of pure singular phase of longer alkyl chain $d \mathrm{~A}$. Displacement of $\mathrm{V}^{\bullet+}$ of shorter chain from the condensed phase and occupation of thus formed vacant area by $\mathrm{V}^{\bullet+}$ of longer chain takes place. This place exchange should accompany an electron transfer from outgoing $\mathrm{V}^{\bullet+}$ to incoming $\mathrm{V}^{2+}$ (Fig. 7-B). This is an energetically downhill process, because oxidation potential of $\mathrm{V}^{\bullet+}$ of shorter one is more negative than reduction potential of $\mathrm{V}^{2+}$ of longer one (Table 1). Note that such a displacement-exchange process, yet electron transfer is not necessarily accompanied, has been observed not only on an HOPG electrode for an anhydride + ester binary system [24] but even on a Au electrode for a binary SAM of adamantane thiolate and alkanethiol [35].

In type 2 (mixture of $d \mathrm{~A}-m+b \mathrm{C}-n$ with $m=n$ ), when the solution content of $b C-n$ is more than half, almost pure phase of it cover the entire surface (Fig. 4). The increase of $d \mathrm{~A}-m$ content in solution results in the well mixed condensed phase. This fact reveals that as long as an extent of similarity in molecular shape and size is satisfied, the presence of the additive $-\mathrm{COOH}$ group does not cause phase separation.

In type 3 (mixture of $d \mathrm{~A}-m+b \mathrm{C}-n$ with $|m-n|=3$ ), phase separation into two domains takes place clearly for $d \mathrm{~A}-7+b \mathrm{C}-4$ mixture in a certain range of solution composition (Fig. 5) but less clearly for $d \mathrm{~A}-4+b \mathrm{C}-7$ (Fig. 6). In the latter combination, we tentatively interpreted that this combination has high compatibility of 
reduced forms of $d \mathrm{~A}-4+b \mathrm{C}-7$ on the HOPG surface as described in section 3.4.2, resulting in one merged anodic peak in the range of $x_{\mathrm{A} 4}=0.99-0.9984$ (Fig. 6). There is another possibility that, because of the occurrence of rapid substitution of $d \mathrm{~A}-4$ for $b C-7$ :

$$
\mathrm{V}^{\bullet+}(b \mathrm{C}-7)_{\text {surface }}+\mathrm{V}^{2+}(d \mathrm{~A}-4)_{\text {solution }} \rightarrow \mathrm{V}^{2+}(b \mathrm{C}-7)_{\text {solution }}+\mathrm{V}^{\bullet+}(d \mathrm{~A}-4)_{\text {surface }}
$$

only one anodic peak at the same potential as the reoxidation potential of pure $\mathrm{V}^{\bullet+}(d \mathrm{~A}-4)_{\text {surface }}$ phase is observed. This substitution involves an electron transfer process from $\mathrm{V}^{\bullet+}(b \mathrm{C}-7)_{\text {surface }}$ to $\mathrm{V}^{2+}(d \mathrm{~A}-4)_{\text {solution. Th }}$ This process is, however, energetically uphill and thus unlikely. To explain the experimental results, the oxidation potential of $\mathrm{V}^{\bullet+}(b \mathrm{C}-7)$ should be largely shifted to more negative in the coexistence of $\mathrm{V}^{\bullet+}(d \mathrm{~A}-4)$ than in its pure condensed phase. This shift may eventually realize simultaneous oxidation processes of $d \mathrm{~A}-4$ and $b \mathrm{C}-7$ at the same potential, exhibiting a single anodic peak in $\mathrm{CV}$ at $x_{\mathrm{A} 4} \geq 0.99$. To attain such a shift, $\mathrm{V}^{\bullet+}(b \mathrm{C}-7)_{\text {surface }}$ and $\mathrm{V}^{\bullet+}(d \mathrm{~A}-4)_{\text {surface }}$ should be well mixed. This is equivalent to the first interpretation that this combination has high compatibility.

In addition, it is noteworthy for type 3 mixture that the minimum fraction of $d \mathrm{~A}-m$ necessary to prevent $b C$ - $n$ from its full surface coverage of a pure phase is $c a .0 .004$ for $d \mathrm{~A}-7+b \mathrm{C}-4$ and $c a .0 .8$ for $d \mathrm{~A}-4+b \mathrm{C}-7$. This is due to either or both very high rigidness of the pure $b \mathrm{C}-7$ phase and weak ability of $d \mathrm{~A}-4$ to destroy the pure phase of others.

Coexistence of two phase-separated domains was observed for the mixtures of two $d$ As with more than two methylene difference as well as those of the type $3(d \mathrm{~A}-7+$ $b \mathrm{C}-4)$, though it appeared only in a narrow range of the mixing ratio as a transitory state. The molar fraction range that gives rise to phase-separated two domains is also of significance. The width of the range $\left(\Delta x_{\mathrm{s}}\right)$ is approximately 0.037 for both $d \mathrm{~A}-6+$ $d \mathrm{~A}-4$ and $d \mathrm{~A}-7+d \mathrm{~A}-4$ mixtures. However, $\Delta x_{\mathrm{s}}$ for $d \mathrm{~A}-7+d \mathrm{~A}-5$ mixture is much greater (0.08). When the difference of the adsorption energy of two individual components is small, the lateral intermolecular interaction could dominantly determine the state of the phase. Therefore, difference of the adsorption energy may be relatively 
small for $d \mathrm{~A}-7+d \mathrm{~A}-5$ mixture and large for $d \mathrm{~A}-7+d \mathrm{~A}-4$ mixture. At moment, we cannot comment on whether it is due to odd-even effect in the number of methylene carbons or not. The value of $\Delta x_{\mathrm{s}}$ is $c a .0 .1$ for $d \mathrm{~A}-7+b \mathrm{C}-4$ mixture. Based on the transition potential in $\mathrm{CV}$, one of the two phases is almost pure $b \mathrm{C}-4$ phase and other is $d$ A-7-enriched phase with small amount of dissolved $b C-4$. Restricted thermodynamic compatibility and similar rigidness of the two phases may be effective to enlarge $\Delta x_{\mathrm{s}}$. We also found an explicit tendency that the pure phase of bis-carboxylated viologen, being once initiated to be created, does not allow strictly the contamination with others, while di-alkyl viologen exhibits high tolerance. The phase with hydrogen bonding network tends to expel dialkyl viologen. A possible exception of this is the case of $d \mathrm{~A}-4+b \mathrm{C}-7$ as discussed above. As demonstrated by the results in alkaline solution, the deprotonated carboxylate form of $b \mathrm{C}$ has no chance to condense to its pure phase.

For the mixtures exhibiting phase separation, it would be meaningful to discuss the correlation between the molar fraction in the solution phase and that in the condensed phase on the HOPG surface. However, we discard this analysis because the interval of molar fraction showing the phase separation is very narrow and the correlation is time dependent due to the transitory nature of the phase separated state.

In the mixtures of two $d$ As with chain length difference of two or more methylene groups, $d \mathrm{~A}-4+b \mathrm{C}-4, d \mathrm{~A}-7+b \mathrm{C}-7$, and $d \mathrm{~A}-7+b \mathrm{C}-4$, the behavior of the viologen originally possessing less negative reduction potential is concerned. At very low content of the viologen, it cannot start condensation, whereas the majority viologen can do at more negative potentials. As long as only the reduction potential is considered, the viologen originally possessing less negative reduction potential could be first condensed. To mention few examples, in the mixture of $d \mathrm{~A}-6+d \mathrm{~A}-4$, when the molar fraction of $d \mathrm{~A}-6$ in the solution is lower than 0.01 , cathodic peak representing condensation of $d A-6$ was not observed in the course of cathodic potential scan, even though the reduction potential of $d \mathrm{~A}-6$ is less negative than that of $d \mathrm{~A}-4$ (Fig. 3-A). Another example has been described in 3.4.1. This would be due to too low concentration to create a nucleus that can start growth or due to the adsorption blocking 
by coexisting majority partner in the mixture (Fig. 7-C).

Because the phase-separated state observed is a transitory state, what was observed in this work is neither equilibrium state nor steady state. Exchange with solution species should be taken into account as shown in Fig. 7-B. Therefore, deeper understanding of the kinetics is highly important. To that purpose, especially to draw a quantitative kinetic model, we need to know exactly the molecular alignment structure of the condensed phase on the electrode surface as a due course. We are, therefore, currently preparing in situ scanning probe microscopic measurements to directly see the 2D molecular organization structure, distribution of the domains, and their time evolution.

\section{Conclusion}

Formation of a viologen radical cation condensed phase through $2 \mathrm{D}$ molecular organization process on an HOPG electrode surface in binary viologen solutions was described using the results of the measurements of cyclic voltammograms for ten binary combination mixtures. In the case of the mixtures of di-alkyl viologens, transitory phase separation was observed when the alkyl chain length difference is two or more methylene units. The phase separated state was found to be eliminated spontaneously through exchange between adsorbed to solution species accompanied by electron transfer process. The molar ratio range showing the phase separation is determined by lateral intermolecular interaction energy in the condensed phase as well as adsorption energy. A well mixed phase formation takes place for the mixture of a di-alkyl viologen and a bis-carboxylated viologen if their methylene chain lengths are the same. The presence of terminal carboxylic acid group is not the factor to facilitate phase separation. Bis-carboxylated viologen tends to form its pure phase, whereas di-alkyl viologen can allow contamination of its condensed phase with the other. The full occupation of the HOPG surface by the condensed phase of viologen, which can first be 
reduced in the course of the negative potential scan, does not always occur. In the mixture of longer di-alkyl viologen and shorter bis-carboxylated viologen, both blocking and triggering of the condensation of di-alkyl viologen by bis-carboxylated viologen were observed. In all the cases, tendency to convergence into the thermodynamically most stable state was observed. Importance of clarification of 2D molecular organization structures of the most stable phase and transitory phase is pointed out. Quantitative understanding of adsorption and intermolecular interaction energies are required in the future study.

\section{Acknowledgements}

This work was financially supported in part by a Grant-in-Aids for Scientific Research B (no. 16350077 for T. S.) from the Ministry of Education, Culture, Sports, Science, and Technology of Japan Government.

\section{References}

[1] N.J. Tao, in: J. Lipkowski, P.N. Ross (Eds.), Imaging of Surface and Interfaces (Frontier of Electrochemistry, Vol. 5), Wiley-VCH, New York, 1999, pp. 211-248.

[2] T. Dretschkow, Th. Wandlowski, in: K. Wandelt, S. Thurgate (Eds.), Solid-Liquid Interfaces, Topics Appl. Phys. 85, Springer-Verlag, Berlin, 2003, pp. 259-321.

[3] T. Wandlowski, in: A.J. Bard, M. Stratmann (Eds.), Encyclopedia of Electrochemistry Vol. 1, Wiley-VCH, Weinheim, 2003, pp. 383-467.

[4] C. Prado, I. Navarro, M. Rueda, H. Rrançois, C. Buess-Herman, J. Electroanal. Chem. 500 (2001) 356-364.

[5] H. Striegler, D. Krznaric, D.M. Kolb, J. Electroanal. Chem. 532 (2002) 227-235.

[6] Th. Wandlowski, K. Ataka, D. Mayer, Langmuir 18 (2002) 4331-4341. 
[7] S. Pronkin, Th. Wandlowski, J. Electroanal. Chem. 550-551 (2003) 131-147.

[8] F. Kitamura, T. Ohsaka, K, Tokuda, J. Electroanal. Chem. 347 (1993) 371-381.

[9] J.I. Millán, R. Rodríguez-Amaro, J.J. Ruiz, L. Camacho, J. Phys. Chem. B 103 (1999) 3669-3676.

[10] K. Arihara, F. Kitamura, K. Nukanobu, T. Ohsaka, K. Tokuda, J. Electroanal. Chem. 473 (1999) 138-144.

[11] T. Sagara, S. Tanaka, Y. Fukuoka, N. Nakashima, Langmuir 17 (2001) 1620-1629.

[12] L. Gómez, J.J. Ruiz, L. Camacho, R. Rodríguez-Amaro, J. Electroanal. Chem. 564 (2004) 179-183.

[13] T. Sagara, S. Tanaka, K. Miuchi, N. Nakashima, J. Electroanal. Chem. 524-525 (2002) 68-76.

[14] T. Sagara, K. Miuchi, J. Electroanal. Chem. 567 (2004) 193-202.

[15] T. Sagara, Y. Fujihara, T. Tada, J. Electrochem. Soc. 152 (2005) E239-246.

[16] D.-T. Pham, K. Gentz, C. Zörlein, N. T. M. Hai, S.-L. Tsay, B. Kirchner, S. Kossmann, K. Wandelt, P. Broekmann, New J. Chem. 30 (2006) 1439-1451.

[17] Y. Tanaka, T. Sagara, Bull. Chem. Soc. Jpn. 80 (2007) 1511-1517.

[18] Y. Tanaka, T. Sagara, Electrochem. Commun. 9 (2007) 741-746.

[19] D. Hobara, M. Ota, S. Imabayashi, K. Niki, T. Kakiuchi, J. Electroanal. Chem. 444 (1998) 113-119.

[20] T. Kakiuchi, K. Sato, M. Iida, D. Hobara, S. Imabayashi, K. Niki, Langmuir, 16 (2000) 7238-7244.

[21] T. Kakiuchi, M. Iida, N. Gon, D. Hobara, S. Imabayashi, K. Niki, Langmuir 17 (2001) 1599-1603.

[22] D. Hobara, T. Kakiuchi, Electrochem. Commun. 3 (2001) 154-157.

[23] B. Xu, S. Yin, C. Wang, Q. Zeng, X. Qiu, C. Bai, Surf. Interface Anal., 32 (2001) 245-249.

[24] K.E. Plass, A.J. Matzger, Chem. Commun. 2006, 3486-3488.

[25] F. Tao, J. Goswami, S.L. Bernasek. J. Phys. Chem. B 110 (2006) 19562-19569.

[26] S. De Feyter, F. C. De Schryver, J. Phys. Chem. B 109 (2005) 4290-4302. 
[27] J.V. Barth, Annu. Rev. Phys. Chem. 58 (2007) 375-407.

[28] H. Gerischer, R. McIntyre, D. Scherson, W. Storck, J. Phys. Chem. 91 (1987) 1930-1935.

[29] T. Sagara, M. Fukuda, N. Nakashima, J. Phys. Chem. B 102 (1998) 521-527.

[30] C.A. Widrig, C. Chung, M.D. Porter, J. Electroanal. Chem. 310 (1991) 335-359.

[31] T. Kakiuchi, H. Usui, D. Hobara, M. Yamamoto, Langmuir 18 (2002) 5231-5238.

[32] K. E. Heusler, Electrochim. Acta 41 (1996) 411-418.

[33] T. Grygar, F. Marken, U. Schröder, F. Scholz, Collect. Czech. Chem. Commun. 67 (2002) 163-208.

[34] A.V. Shevade, J. Zhou, M.T. Zin, S. Jiang, Langmuir 17 (2001) 7566-7572.

[35] A.A. Dameron, L.F. Charles, P.S. Weiss, J. Am. Chem. Soc. 127 (2005) 8697-8704. 
Table 1. List of characteristic CV data for six viologens singular systems at a sweep rate of $80 \mathrm{mV} \mathrm{s}^{-1}$ at $\mathrm{pH}$ around 2.0 at $23^{\circ} \mathrm{C}$.

\begin{tabular}{rcccc}
\hline viologen & $E_{\mathrm{pa}} / \mathrm{mV}$ & $E_{\mathrm{pc}} / \mathrm{mV}$ & $E_{1 / 2, \mathrm{a}} / \mathrm{mV}$ & $\Delta E_{\mathrm{p}} / \mathrm{mV}$ \\
\hline$d \mathrm{~A}-4$ & -426 & -448 & -437 & 22 \\
$d \mathrm{~A}-5$ & -382 & -407 & -395 & 25 \\
$d \mathrm{~A}-6$ & -333 & -373 & -353 & 40 \\
$d \mathrm{~A}-7$ & -295 & -346 & -321 & 51 \\
$b \mathrm{C}-4$ & -482 & -569 & -526 & 87 \\
$b \mathrm{C}-7$ & -333 & -523 & -428 & 190 \\
\hline
\end{tabular}


Molar fractions of the solution composition ( $x_{\mathrm{A} 7}$ : molar fraction of $\left.d \mathrm{~A}-7\right)$ are given at the right of $\mathrm{CV}$ curves. Figure in $(\mathrm{C})$ is reprinted from ref. [18] with permission from Elsevier.

Fig. 5. (A) CVs for binary mixture of $d \mathrm{~A}-7+b \mathrm{C}-4$ at an $\mathrm{HOPG}$ electrode at a potential sweep rate of $80 \mathrm{mV} \mathrm{s}^{-1}$. Molar fractions of the solution composition $\left(x_{\mathrm{A} 7}\right.$ : molar fraction of $d \mathrm{~A}-7$ ) are given at the right of $\mathrm{CV}$ curves. (B) Plots of $\mathrm{CV}$ peak potentials for binary mixture of $d \mathrm{~A}-7+b \mathrm{C}-4$ against $\log x_{\mathrm{A} 7}$. Closed and open marks represent, respectively, cathodic and anodic peak potentials.

Fig. 6. (A) CVs for binary mixture of $d \mathrm{~A}-4+b \mathrm{C}-7$ at an HOPG electrode at a potential sweep rate of $80 \mathrm{mV} \mathrm{s}^{-1}$. Molar fractions of the solution composition $\left(x_{\mathrm{A} 4}\right.$ : molar fraction of $d \mathrm{~A}-4$ ) are given at the right of $\mathrm{CV}$ curves. (B) Plots of CV peak potentials for binary mixture of $d \mathrm{~A}-4+b \mathrm{C}-7$ against $x_{\mathrm{A} 4}$. Closed and open marks represent, respectively, cathodic and anodic peak potentials.

Fig. 7. A cartoon representing schematically the typical situations for binary condensed phase formation process and time evolution. $\mathrm{X}$ stands for the viologen possessing less negative reduction potential and $\mathrm{Y}$ does that possessing more negative reduction potential.

Case A: well-mixed condensed phase formation (eg. $d \mathrm{~A}+d \mathrm{~A}$ with alkyl chain length difference is one methylene unit, $d \mathrm{~A}+b \mathrm{C}$ with the same number of methylene units).

Case B: transitory phase-separated two domain formation and afterward exchange processes (eg. $d \mathrm{~A}+d \mathrm{~A}$ with alkyl chain length difference is 2 or 3 methylene units, $d \mathrm{~A}$ $+b \mathrm{C}$ with different chain length).

Case C: very small molar fraction of X. Arrow (a) in the figure is valid for $d \mathrm{~A}+d \mathrm{~A}$ mixture. 


\section{Legends of Scheme and Figures}

Scheme 1. Six viologen molecules used in the work with their abbreviations (upper) and binary combinations examined (lower). The three types of mixing indexed in the text are shown by combination lines: solid line for type 1 (mixture of two $d A s$ ), doubled solid line for type 2 (mixture of $d \mathrm{~A}$ and $b \mathrm{C}$ with same number of methylene units), and dashed line for type 3 (cross mixture of $d \mathrm{~A}$ and $b \mathrm{C}$ ). Combination of dotted line is out of scope of this work.

Fig. 1. Series of CVs for binary mixtures of $d \mathrm{~A}-\mathrm{m}+d \mathrm{~A}-(m-1)$ for $m=5(\mathrm{~A}), m=6$ (B), and $m=7$ (C) at an HOPG electrode at a potential sweep rate of $80 \mathrm{mV} \mathrm{s}^{-1}$. Molar fractions of the solution composition ( $x_{\mathrm{m}}$ : molar fraction of longer chain viologen) are given at the right of CV curves.

Fig. 2. Plot of peak potentials (open circles for $E_{\mathrm{pa}}$ and closed circles for $E_{\mathrm{pc}}$ ) of $\mathrm{CVs}$ for the mixture of $d \mathrm{~A}-6+d \mathrm{~A}-5$ at an $\mathrm{HOPG}$ electrode at a potential sweep rate of $80 \mathrm{mV} \mathrm{s}^{-1}$.

Fig. 3. Series of CVs for binary mixtures of $d \mathrm{~A}-6+d \mathrm{~A}-4(\mathrm{~A})$ and $d \mathrm{~A}-7+d \mathrm{~A}-5(\mathrm{~B})$ at an HOPG electrode at a potential sweep rate of $80 \mathrm{mV} \mathrm{s}^{-1}$. Molar fractions of the solution composition ( $x_{\mathrm{m}}$ : molar fraction of longer chain viologen) are given at the right of CV curves.

Fig. 4. (A) CVs for binary mixture of $d \mathrm{~A}-4+b \mathrm{C}-4$ at an HOPG electrode at a potential sweep rate of $80 \mathrm{mV} \mathrm{s}^{-1}$. Molar fractions of the solution composition $\left(x_{\mathrm{A} 4}\right.$ : molar fraction of $d \mathrm{~A}-4$ ) are given at the right of $\mathrm{CV}$ curves. (B) Plots of CV peak potentials for binary mixture of $d \mathrm{~A}-4+b \mathrm{C}-4$ against $x_{\mathrm{A} 4}$. Closed and open marks represent, respectively, cathodic and anodic peak potentials. (C) CVs for binary mixture of $d \mathrm{~A}-7+b \mathrm{C}-7$ at an HOPG electrode at a potential sweep rate of $80 \mathrm{mV} \mathrm{s}^{-1}$. 


\section{Viologen molecules}

$$
\begin{aligned}
& \mathrm{H}_{3} \mathrm{C}-\left(\mathrm{CH}_{2}\right)_{m-1}-\mathrm{Nr}^{+} \\
& d A-m, m=4,5,6,7
\end{aligned}
$$

$$
\begin{aligned}
& \text { HOOC- }\left(\mathrm{CH}_{2}\right)_{n} \mathrm{Nr}^{+-} \mathrm{N}^{-} \\
& \text {bC- } n, n=4,7
\end{aligned}
$$

\section{Binary combinations}

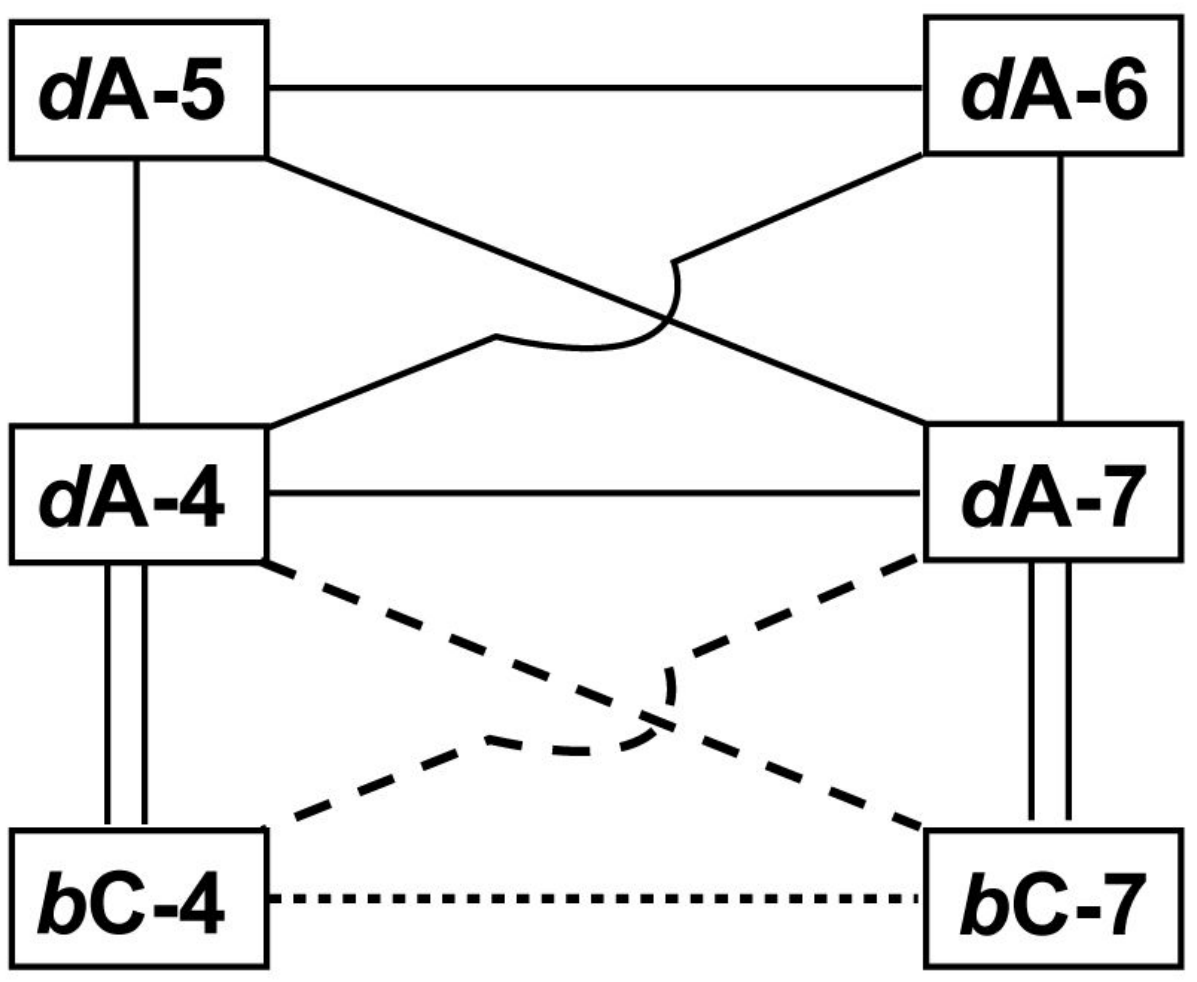



(A) $d \mathrm{~A}-5+d \mathrm{~A}-4$
$x_{5}$
(B) $d \mathrm{~A}-6+d \mathrm{~A}-5$
(C) $d \mathrm{~A}-7+d \mathrm{~A}-6$
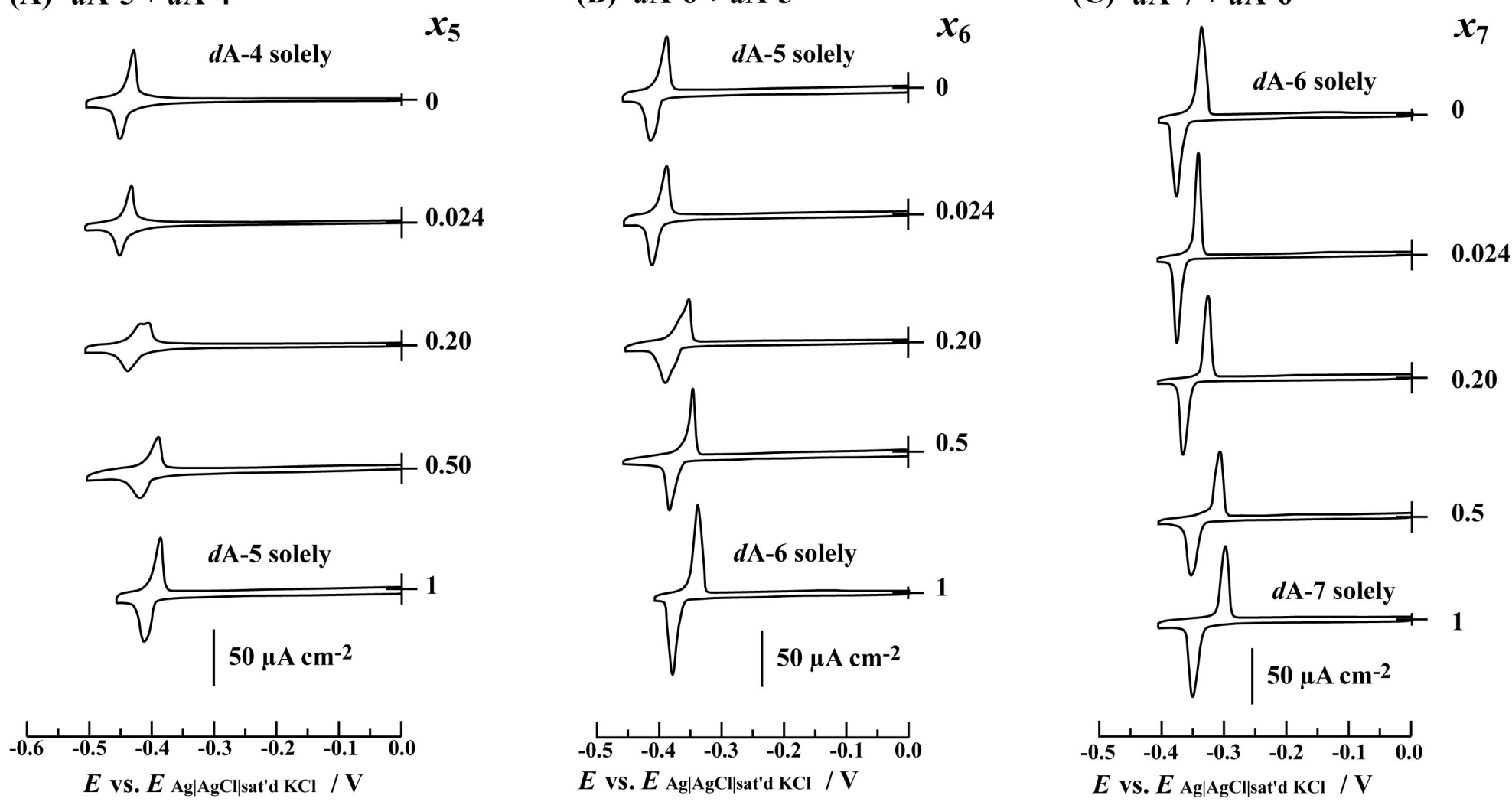
$E$ vs. $E$ Ag|AgCl|sat'd KCl / V

Figure 1 


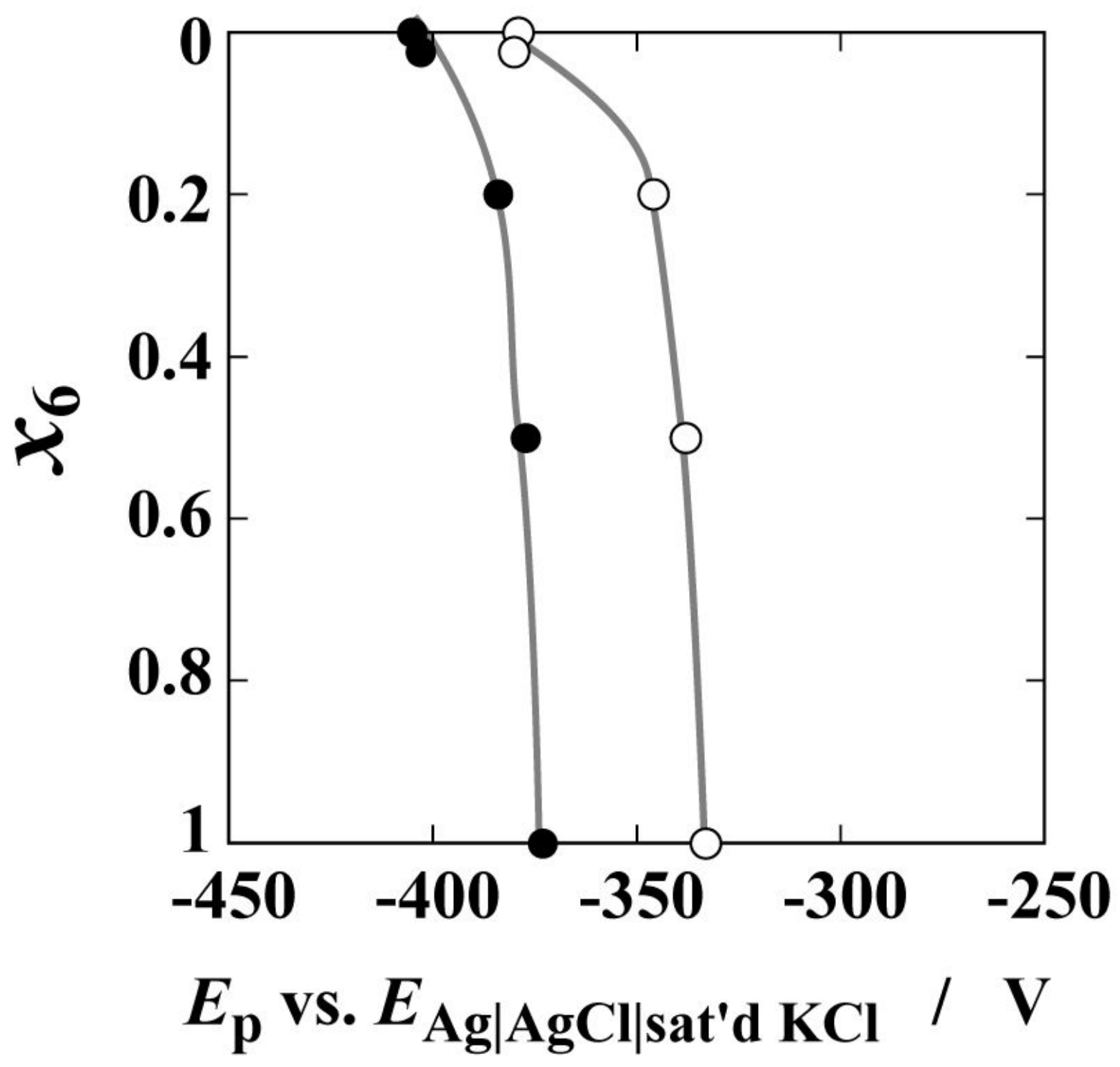

Figure 2 
(A) $d \mathrm{~A}-6+d \mathrm{~A}-4$

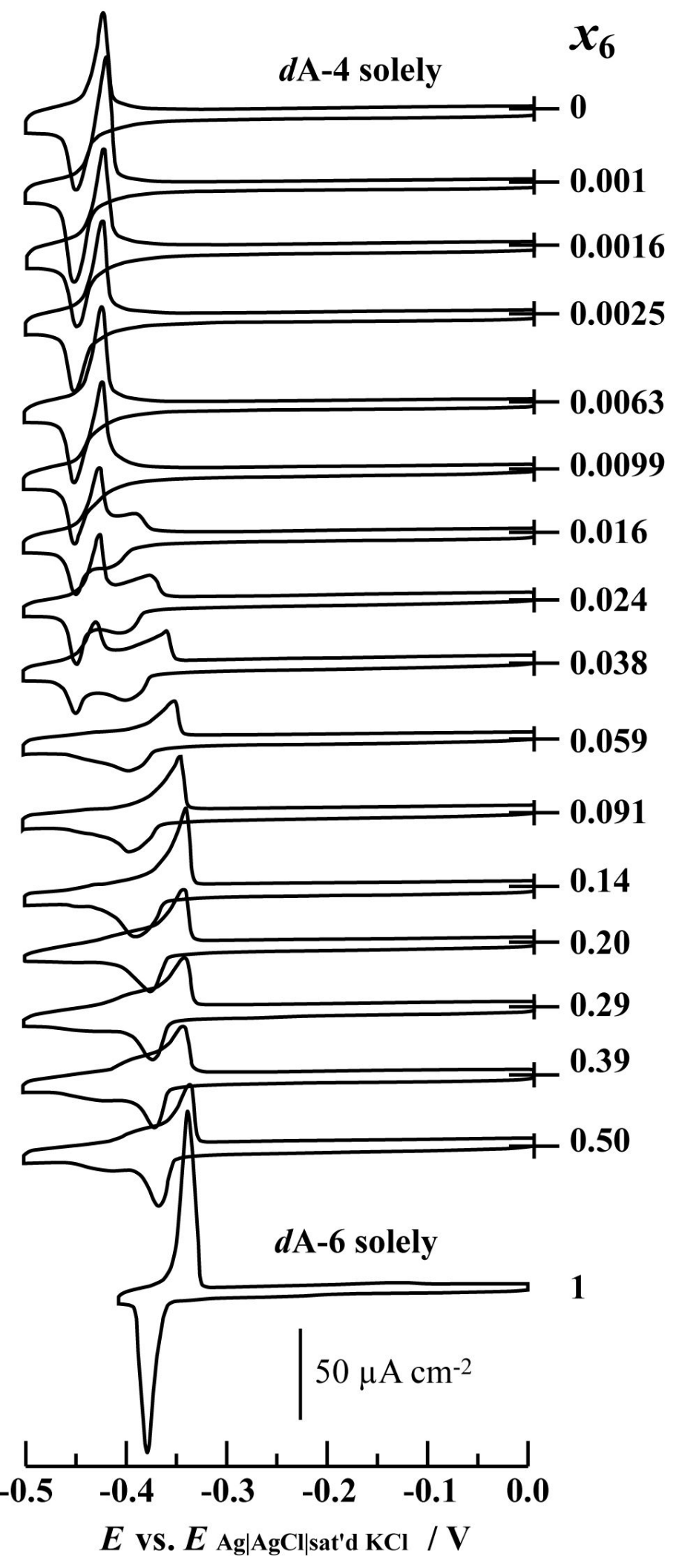

(B) $d \mathrm{~A}-7+d \mathrm{~A}-5$

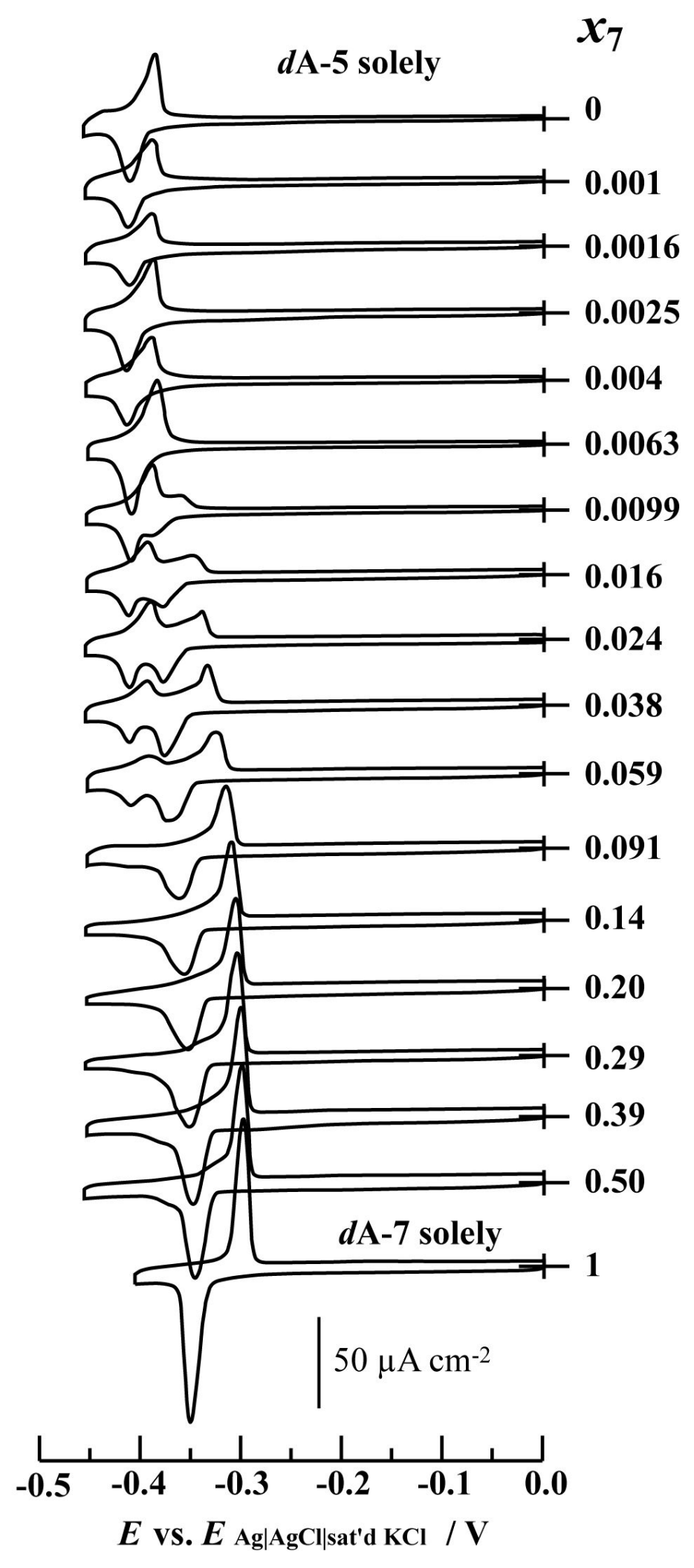


(A) $d \mathrm{~A}-4+b \mathrm{C}-4$

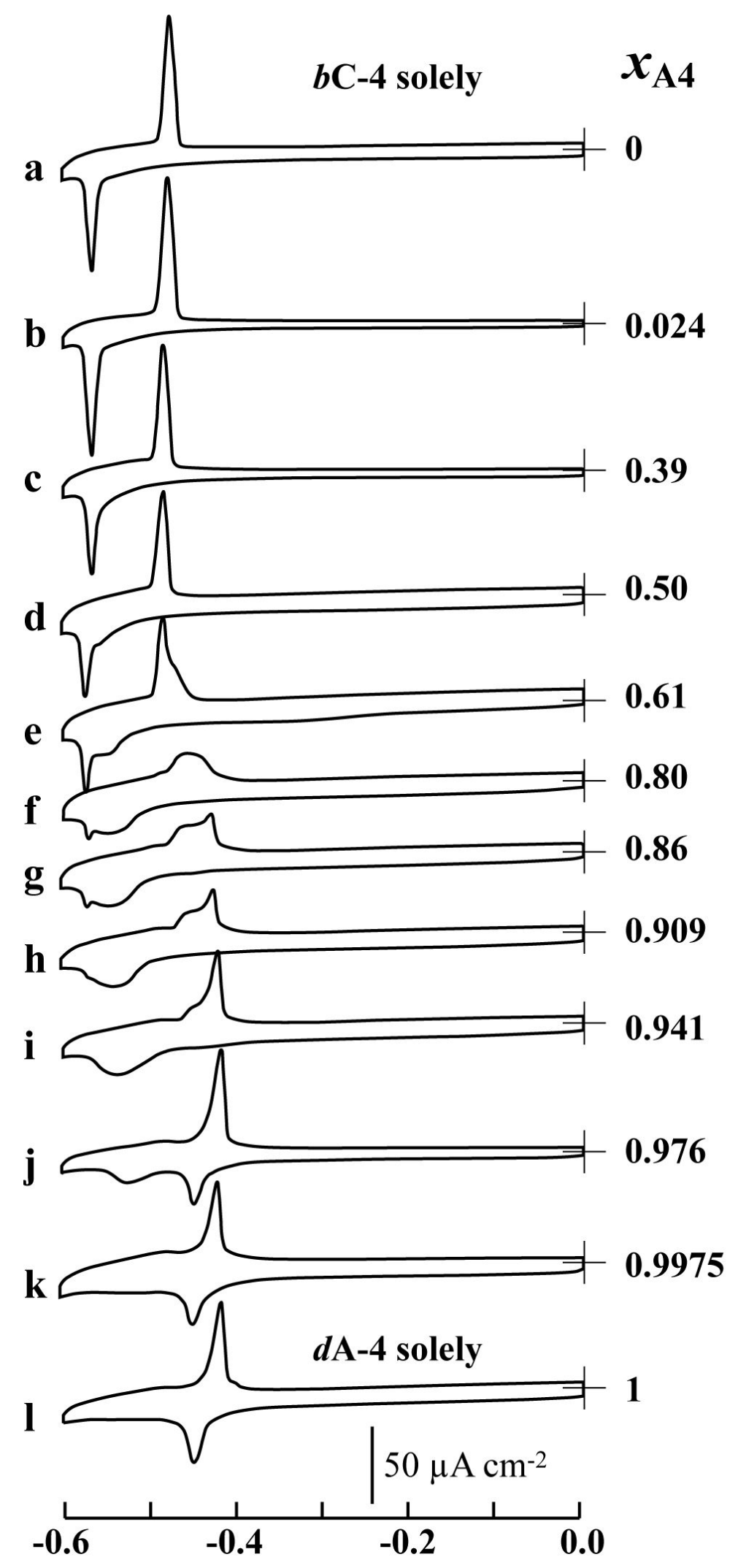

E vs. $E$ Ag|AgCl|sat'd KCl / V
(B) $d \mathrm{~A}-4+b \mathrm{C}-4$

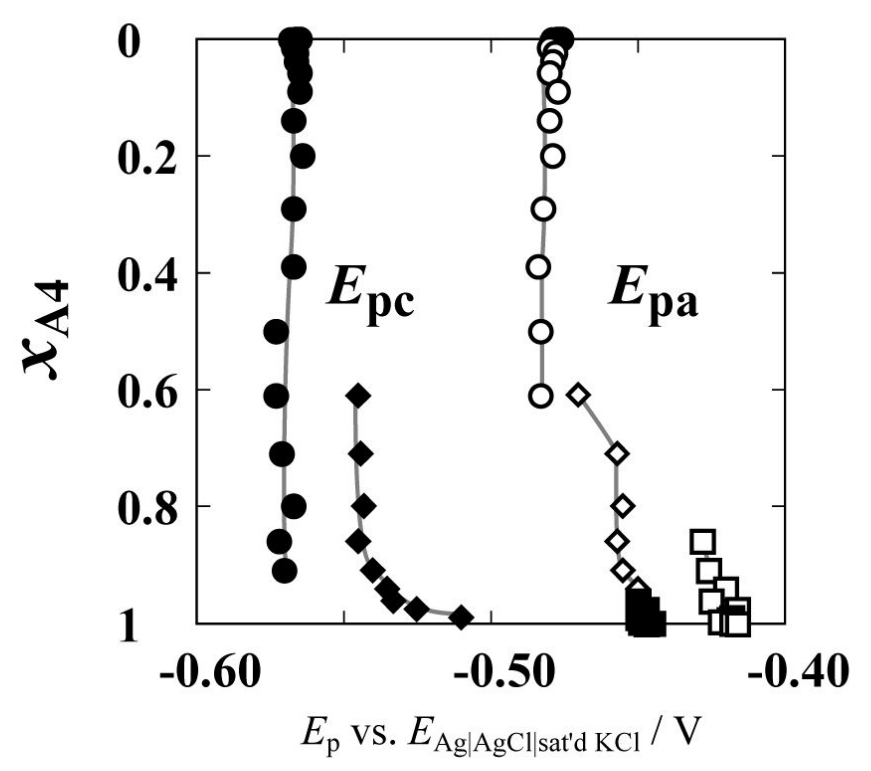

(C) $d \mathrm{~A}-7+b \mathrm{C}-7$

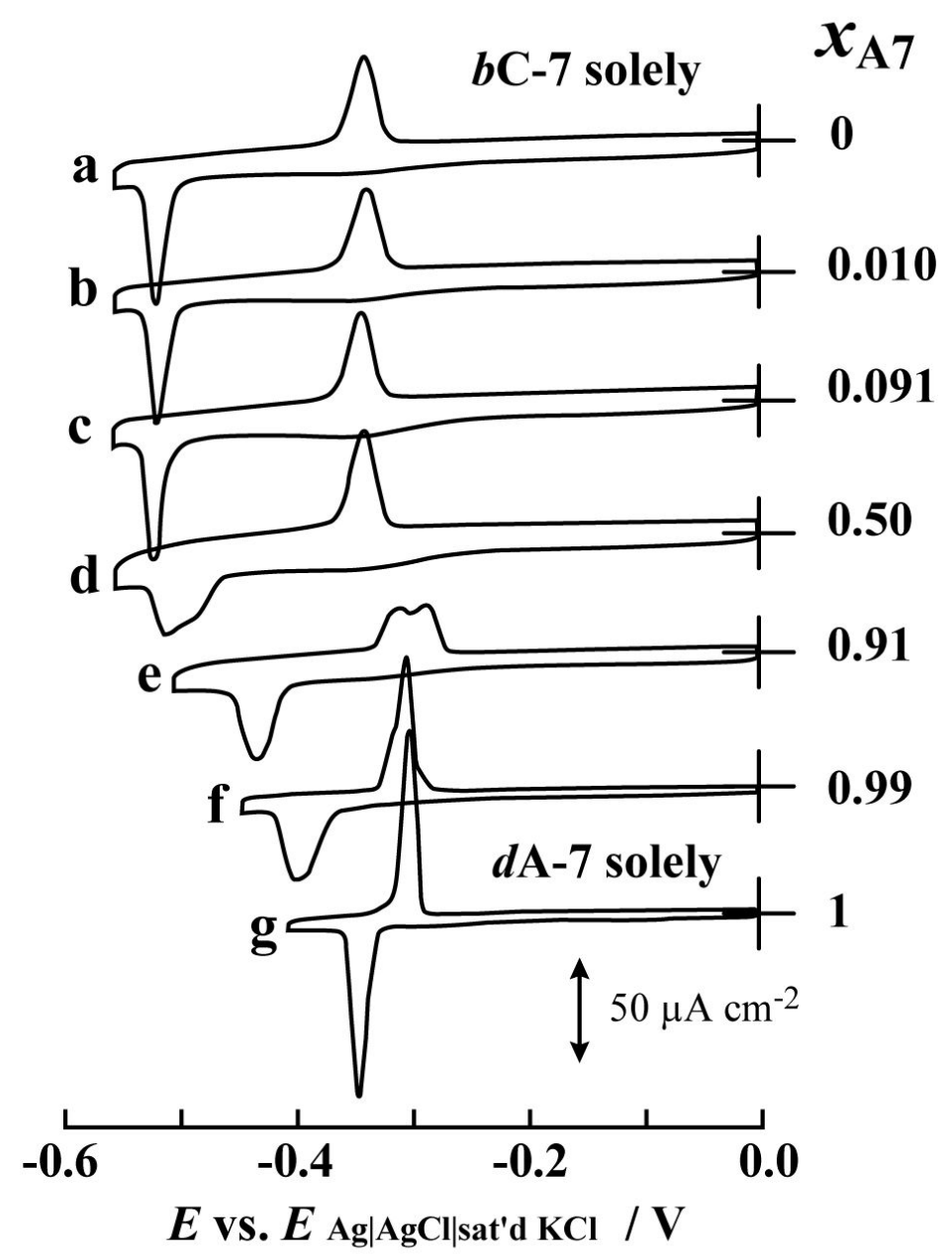


(A) $d \mathrm{~A}-7+b \mathrm{C}-4$

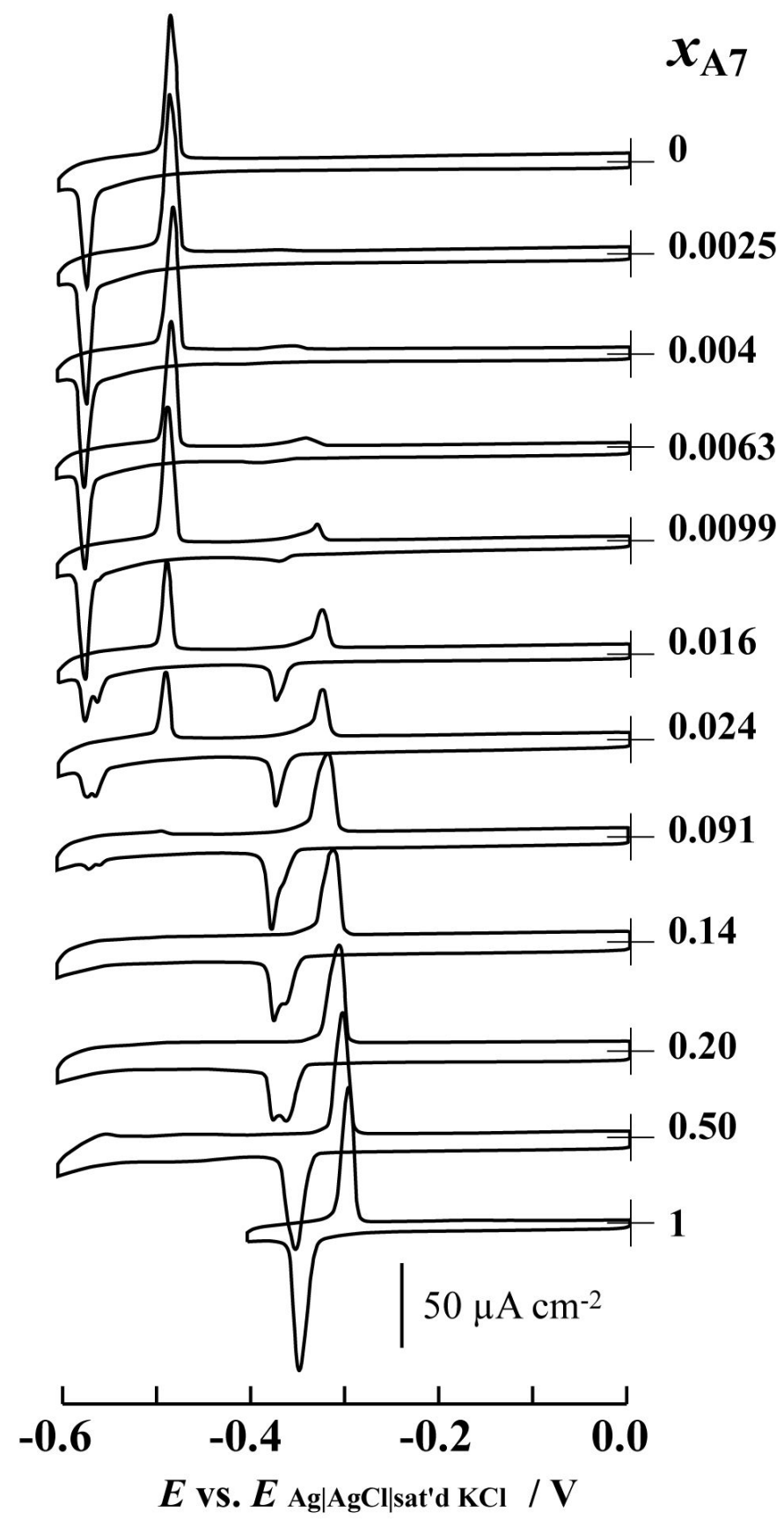

(B)

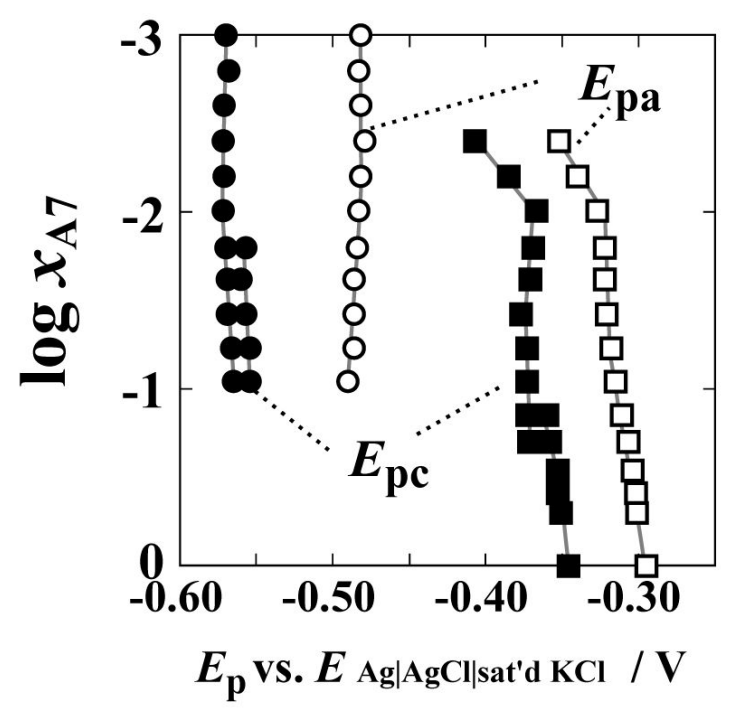

Figure 5 
(A) $d \mathrm{~A}-4+b \mathrm{C}-7$

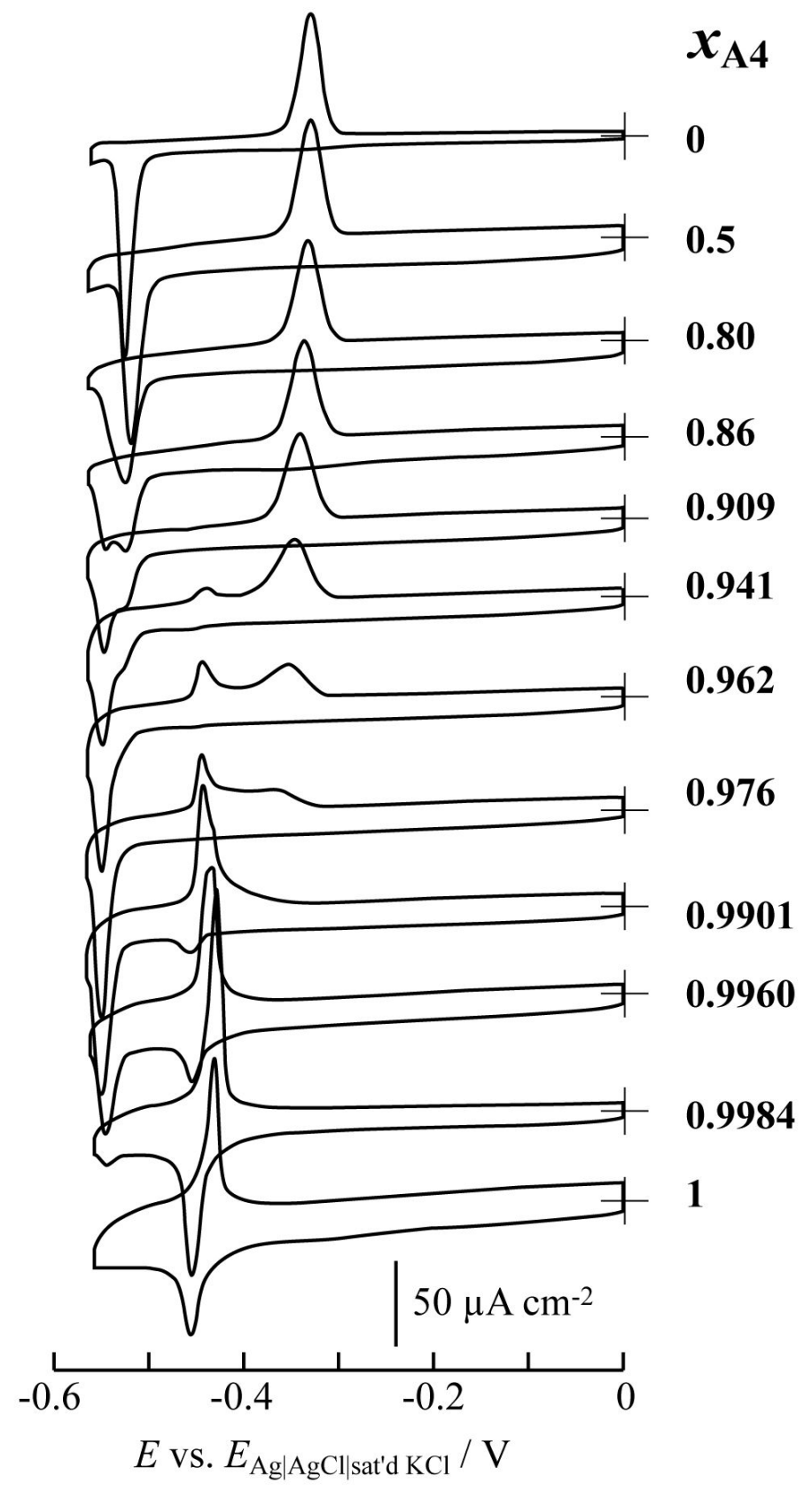

(B)

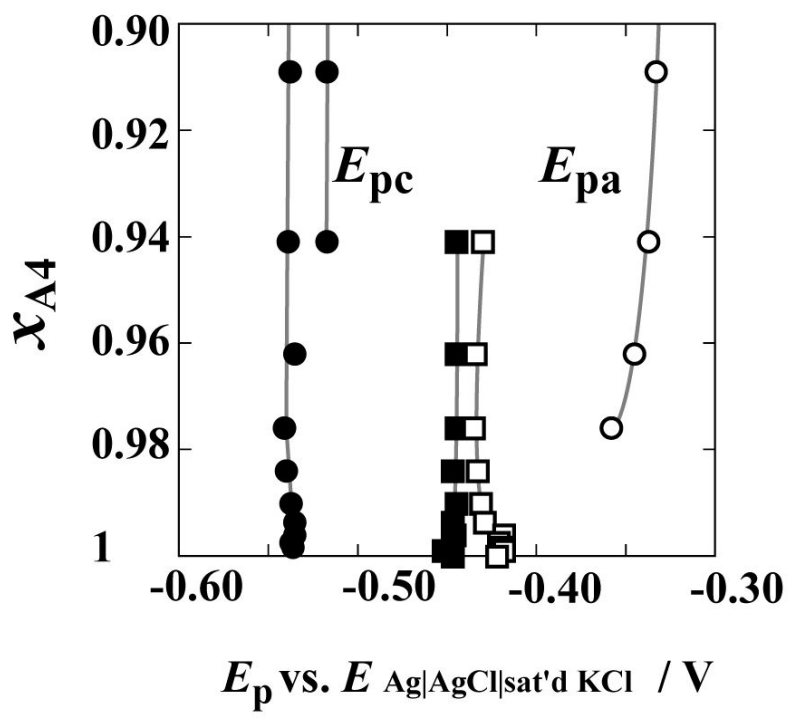

Figure 6 
Case A

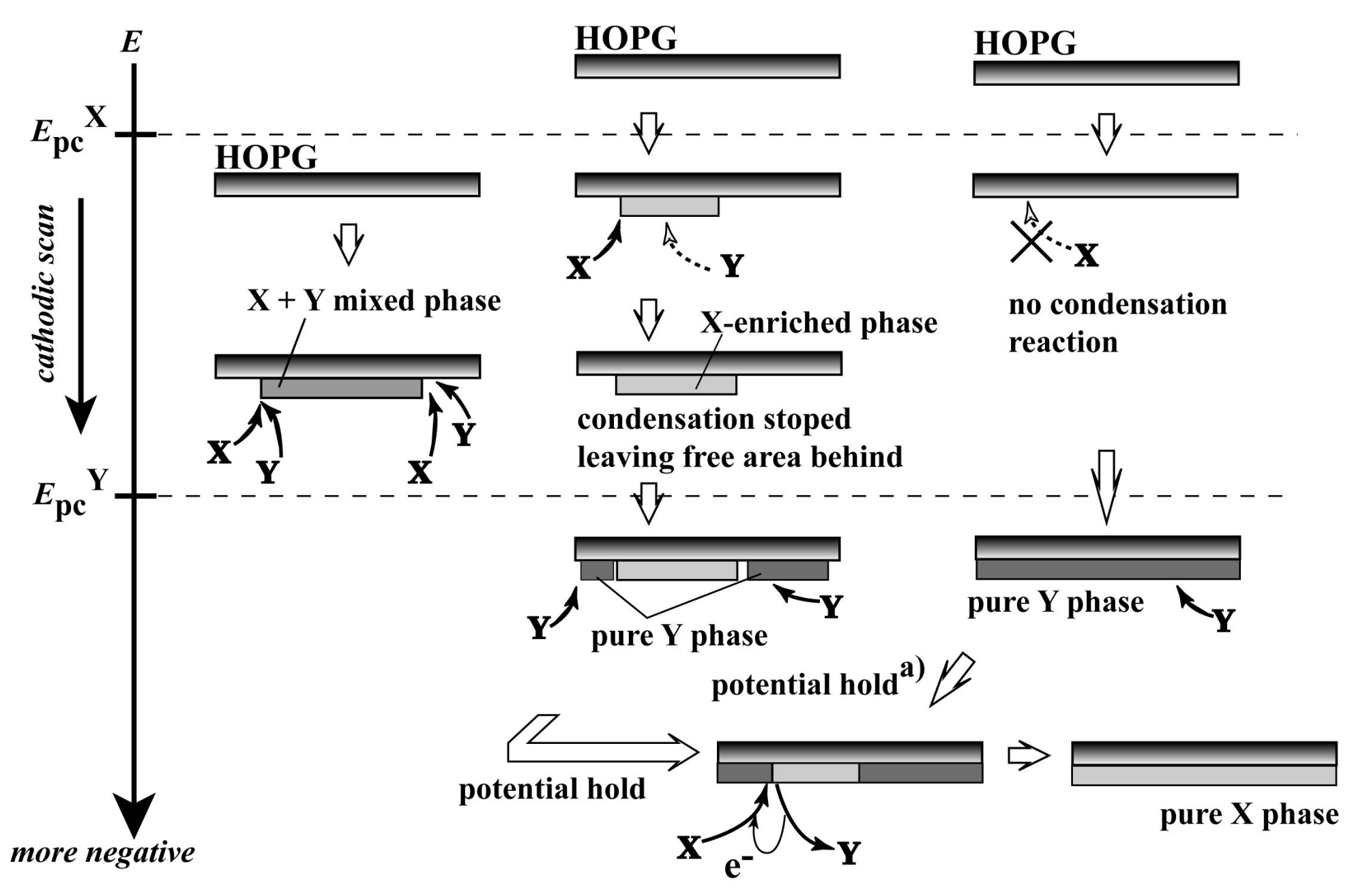

Case B

Case C

HOPG

HOPG 